\title{
Viscous flow over a chemically patterned surface
}

\author{
J.E. Sprittles* and Y.D. Shikhmurzaev ${ }^{\oplus}$ \\ School of Mathematics, University of Birmingham, Birmingham, B15 2TT, UK.
}

(Dated: October 31, 2018)

\begin{abstract}
The classical fluid dynamics boundary condition of no-slip suggests that variation in the wettability of a solid should not affect the flow of an adjacent liquid. However experiments and molecular dynamics simulations indicate that this is not the case. In this paper we show how flow over a solid substrate with variations of wettability can be described in a continuum framework using the interface formation theory developed earlier. Results demonstrate that a shear flow over a perfectly flat solid surface is disturbed by a change in its wettability, i.e. by a change in the chemistry of the solid substrate. The magnitude of the effect is shown to be proportional to $\cos \theta_{1}-\cos \theta_{2}$ where $\theta_{1}$ and $\theta_{2}$ are the equilibrium contact angles that a liquid-gas free surface would form with the two chemically different parts of the solid surface.

PACS numbers: 68.08.-p, 47.61.-k, 68.43.-h
\end{abstract}

\section{INTRODUCTION}

The flow of liquids over chemically patterned surfaces is an exciting and relatively new area of fluid mechanics with applications in many emerging technologies [1]. Such flows are of particular interest in microfluidics where an increasing surface to volume ratio of liquids means that surface effects become of greater significance [2]. The correct description of the physics at solid-liquid interfaces then becomes imperative to the success of any attempt to model this class of flows [3]. It has been shown that by patterning a substrate with hydrophillic and hydrophobic regions it is possible to confine a liquid to a microchannel [4, 5], to improve the accuracy of droplet deposition [6, 7] or to create a structured film [8]. Alternatively, when a wettability gradient is present, unbalanced surface tension forces can lead to the movement of liquid drops [9, 10].

The effect of variable wettability of the solid substrate on the adjacent flow has been studied theoretically using molecular dynamics simulations [11, 12]. In this approach, variations in wettability are modelled by varying an interaction potential between molecules of the solid and the fluid. The results show that a change in wettability does affect the flow, most notably producing a component of velocity normal to the solid surface. Given that "wettability" can be introduced as a macroscopic characteristic of a liquid-solid system, one should be able to model the effects discovered by molecular dynamics macroscopically using an appropriate formulation in the framework of continuum mechanics.

In the continuum approximation, molecular properties of the contacting media, as well as molecular length and time scales, do not appear explicitly. Instead, they manifest themselves in the bulk equations and boundary conditions via macroscopic transport coefficients and

\footnotetext{
*Electronic address: sprittlj@maths.bham.ac.uk

${ }^{\dagger}$ Electronic address: Y.D.Shikhmurzaev@bham.ac.uk
}

parameters of constitutive equations. The classical noslip boundary condition leaves no room for incorporating the effects of variable wettability and, as is well known [13, 14], it becomes inadequate for dealing with processes of dynamic wetting where wettability of the solid plays a key role.

A generalisation of no-slip often cited in the literature is the Navier slip condition [15, 16], where slip, i.e. the difference between the tangential velocities of the fluid and the solid, is assumed to be proportional to the tangential stress acting from the fluid on the liquid-solid interface. The coefficient of proportionality is referred to as the 'slip coefficient' or the 'coefficient of sliding friction'. Various expressions for this coefficient have been proposed [17] some of which are based on the results of molecular dynamics simulations [18]. An appropriate choice of the slip coefficient allows one to describe situations in which the characteristic length scales of the flow are small (e.g. nanopores), so that the effects of slip become apparent [19].

The problem arises when the approach based on the Navier slip condition is used to describe flows over solid surfaces with variable wettability, since the existing models provide no conceptual link between slip and wettability. Indeed, as reviewed in $\S 9$ of [20], in the area of dynamic wetting, where the physics of wettability becomes a key factor, the so-called 'slip models' treat the behaviour of the contact angle, which is the macroscopic 'measure' of wettability, and slip, which is used as a boundary condition removing the stress singularity at the moving contact line, as completely independent. On a macroscopic level, any coupling of a formula for the velocity-dependence of the dynamic contact angle and a slip boundary condition will produce a new slip model. Alternatively, linking the slip coefficient (or any other slip condition) to wettability via molecular dynamics simulations negates the advantages of the self-contained and experimentally verifiable continuum modelling.

Applying the no-slip or constant slip coefficient Navier boundary condition at a solid surface implies that the flow of a liquid will not be affected by variations in the 
wettability of an adjacent solid. This is in direct conflict with the conclusions of molecular dynamics simulations [11, 12] that were specifically tailored to investigate this problem. In the present paper we consider flow over a solid surface of variable wettability using a model developed earlier [21] that incorporates wettability in the framework of continuum mechanics from the following perspective. Given that dynamic wetting is, by its very name, a process of creating a new/fresh liquid-solid interface ('a wetted solid surface'), an adequate mathematical model of dynamic wetting must have the physics of interface formation process at its core. The model (hereafter referred to as the interface formation model) has been derived from first principles [21] using methods of irreversible thermodynamics and notably it ensures that all aspects of wettability (contact angle, slip, adsorption) are interrelated. Embedding dynamic wetting in a general physical framework, as a particular case from a broad class of flows with forming/disappearing interfaces, allows one to use the same model, without any ad hoc alterations, to describe other flows from this class [22, 23, 24]. This makes it possible to use the values for material constants determined from completely independent experiments. Comparison with such experiments have confirmed the model's validity and accuracy [21, 25].

We will examine the fully representative case of a plane-parallel shear flow over a smooth solid surface that encounters a transition region between substrates of different wettability.

\section{PROBLEM FORMULATION}

Consider the steady flow of an incompressible Newtonian liquid passing over a stationary flat solid surface. The liquid is driven over the solid by a plane-parallel shear of magnitude $S$ in the far field. We consider the bulk flow to be described by the Navier-Stokes equations

$$
\nabla \cdot \mathbf{u}=0, \quad \rho \mathbf{u} \cdot \nabla \mathbf{u}=-\nabla p+\mu \nabla^{2} \mathbf{u}
$$

where $\mathbf{u}, p, \rho$ and $\mu$ are the fluid's velocity, pressure, density and viscosity, respectively. The boundary conditions on a solid surface of uniform wettability, that follow from the interface formation theory [21] are given by

$$
\begin{gathered}
\mu \mathbf{n} \cdot\left[\nabla \mathbf{u}+(\nabla \mathbf{u})^{*}\right] \cdot(\mathbf{I}-\mathbf{n n})+\frac{1}{2} \nabla \sigma=\beta \mathbf{u} \cdot(\mathbf{I}-\mathbf{n n}), \\
\rho \mathbf{u} \cdot \mathbf{n}=\frac{\rho^{s}-\rho_{e}^{s}}{\tau}, \\
\nabla \cdot\left(\rho^{s} \mathbf{v}^{s}\right)=-\frac{\rho^{s}-\rho_{e}^{s}}{\tau} \\
\left(\mathbf{v}^{s}-\frac{1}{2} \mathbf{u}\right) \cdot(\mathbf{I}-\mathbf{n n})=\alpha \nabla \sigma, \\
\sigma=\gamma\left(\rho_{(0)}^{s}-\rho^{s}\right) .
\end{gathered}
$$

Here $\sigma$ is the surface tension in the 'surface phase', i.e. a microscopic layer of liquid adjacent to the solid surface subject to intermolecular forces from two bulk phases; $\rho^{s}$ is the surface density (mass per unit area) of this layer and $\mathbf{v}^{s}$ is the velocity with which it is transported; $\mathbf{n}$ is the unit vector normal to the solid surface pointing into the liquid; $\mathbf{I}$ is the metric tensor; $(\mathbf{I}-\mathbf{n n})$ is a tensor that extracts the tangential component of a vector, for example $\mathbf{u} \cdot(\mathbf{I}-\mathbf{n n})=\mathbf{u}_{\|}$, where the double line subscript denotes tangential component; $\alpha, \beta, \gamma, \tau, \rho_{(0)}^{s}$ and $\rho_{e}^{s}$ are phenomenological material constants.

The model has previously been discussed in detail (e.g. 21]), so that here we will only briefly recapitulate the meaning of the terms. The surface tension is considered as a dynamic quantity related to the surface density via the equation of state in the 'surface phase' (6) which is taken here in the simplest linear form. The constant $\gamma$ is associated with the inverse compressibility of the fluid whilst $\rho_{(0)}^{s}$ is the surface density corresponding to zero surface tension. As in Gibbs' theory of capillarity, the surface tension itself may be positive or negative depending on whether the solid is hydrophobic or hydrophillic. Gradients in surface tension influence the flow, firstly, via the tangential stress boundary condition (2), i.e. via the Marangoni effect, and, secondly, in (5) by forcing the surface velocity $\mathbf{v}^{\mathbf{s}}$, which is parallel to the solid surface, to deviate from that generated in the surface phase by the outer flow. The constants $\alpha$ and $\beta$ characterise the response of the interface to surface tension gradients and an external torque, respectively; in the simplest variant of the theory both are properties of the fluid and have no relation to the wettability of the solid. Mass exchange between the bulk and surface phases, caused by the possible deviation of the surface density from its equilibrium value $\rho_{e}^{s}$, is accounted for in the boundary condition for the normal component of bulk velocity (3) and in the surface mass balance equation (44). The parameter $\tau$ is the surface tension relaxation time.

One would expect a generalised set of boundary conditions to have the no-slip condition as its limiting case. For the interface formation model this limiting case follows from the limits $\mu /(\beta L), U \tau / L \rightarrow 0$, where $L$ and $U$ are characteristic length and velocity scales of the flow, applied to (2) -(6). Estimates for the phenomenological constants $\beta$ and $\tau$, obtained from experiments on dynamic wetting [25], suggest that, say, for $L \sim 10^{-2} \mathrm{~cm}$, $U \sim 1 \mathrm{~cm} \mathrm{~s}^{-1}$ and $\mu \sim 10 \mathrm{~g} \mathrm{~cm}^{-1} \mathrm{~s}^{-1}$ one has $\mu /(\beta L) \sim$ $10^{-5}$ and $U \tau / L \sim 10^{-6}$. Then for flows which, unlike dynamic wetting, are not associated with infinitesimal length scales, boundary conditions (2)-(6) to leading order in $\mu /(\beta L)$ and $U \tau / L$, reduce to no-slip.

Conditions (22)-(6) were derived using methods of irreversible thermodynamics assuming that the equilibrium surface density $\rho_{e}^{s}$, determined by the wettability of the solid substrate, is a constant, i.e. for a solid surface of a uniform wettability. This constant is related to the equilibrium contact angle that a liquid-gas free surface would form with the solid via the Young equation in the 
following way. If we assume that the equilibrium surface tension of the solid-gas interface is negligible, the Young equation takes the form

$$
\sigma_{s l}=-\sigma_{l g} \cos \theta
$$

where $\sigma_{s l}$ and $\sigma_{l g}$ are the equilibrium surface tensions of the solid-liquid and liquid-gas interfaces and $\theta$ is the equilibrium contact angle that the free surface forms with the solid. Thus, since according to ([6), $\sigma_{s l}=\gamma\left(\rho_{(0)}^{s}-\rho_{e}^{s}\right)$ for a given liquid the Young equation (7) allows one to express $\rho_{e}^{s}$ in terms of $\theta$ (see (19) below).

In order to modify (2) - (6) for a solid of variable wettability we must replace, in (2) and (5)

$$
\nabla \sigma \rightarrow \nabla \sigma+\rho^{s} F^{s}, \text { where } F^{s}=\frac{\gamma \nabla \rho_{e}^{s}}{\rho_{e}^{s}} .
$$

The reaction force $F^{s}$ acts on the liquid-solid interfacial layer from the solid surface and by balancing gradients of the equilibrium surface tension $\sigma_{e}=\sigma\left(\rho_{e}^{s}\right)$, ensures the existence of a state of equilibrium.

Consider the equilibrium surface density of the form

$$
\rho_{e}^{s}=\frac{1}{2}\left(\rho_{1 e}^{s}+\rho_{2 e}^{s}\right)+\frac{1}{2}\left(\rho_{2 e}^{s}-\rho_{1 e}^{s}\right) \tanh (x / l),
$$

where $\rho_{1 e}^{s}$ and $\rho_{2 e}^{s}$ are the equilibrium surface densities in the far field as $x \rightarrow-\infty$ and $x \rightarrow \infty$, respectively; $l$ is the length of the transition region; $x$ is a Cartesian coordinate in the plane of the solid surface. The equilibrium surface density in the form of (9) allows one to investigate the role of a transition region between solids of different wettabilities and, after taking the limit $l / L \rightarrow 0$, find what boundary conditions for the surface variables one should use if this region is modelled as a solid-solid-liquid contact line.

Finally, we will assume that the flow is plane-parallel in the $(x, y)$-plane of a Cartesian coordinate system, the origin of which is at the centre of the transition region, and that it is generated by constant shear of magnitude $S$ in the far field,

$$
\mathbf{u} \rightarrow S \mathbf{i j} \cdot \mathbf{r} \text { as } \mathbf{r} \rightarrow \infty
$$

where $\mathbf{i}$ and $\mathbf{j}$ are unit vectors in the $x$ and $y$ directions and $\mathbf{r}$ is the radius-vector. Equations (1)-10) now completely specify the problem.

It is important to emphasise that in the derivation of the model it is assumed that on the solid-facing side of the solid-liquid interface one has impermeability and noslip. However, it is the velocity on the liquid-facing side of the solid-liquid interface that is the boundary condition for the Navier-Stokes equations (11). In the classical noslip condition it is assumed that there is no difference in velocity between the solid-facing and liquid-facing side of the interface, whereas in the interface formation model, the velocity on the liquid-facing side of the interface is determined by the interaction occurring in the surface phase and between the surface phase and the bulk. As a result, one can expect effective (or 'apparent') slip, i.e. the difference between the velocity on the liquid-facing side of the interface and the velocity of the solid surface, that is, in our case, $\mathbf{u} \cdot(\mathbf{I}-\mathbf{n n}) \neq 0)$ and a non-zero normal component of velocity (a flux in/out of the surface phase, i.e. $\mathbf{u} \cdot \mathbf{n} \neq 0$ ).

It is convenient to non-dimensionalise equations (1)(10) using

$$
U=\mu^{-1} \sigma_{l g}, \quad L=U S^{-1}, \quad P=\mu S, \quad \sigma_{l g}, \quad \rho_{(0)}^{s}
$$

as the scales for velocities, length, pressure, surface tension and surface density. Then in the bulk one has

$$
\nabla \cdot \mathbf{u}=0, \quad \operatorname{Re}(\mathbf{u} \cdot \nabla \mathbf{u})=-\nabla p+\nabla^{2} \mathbf{u} .
$$

whilst on the surface, where we use the notation $u$ and $v$ for tangential and normal components of velocity,

$$
\begin{gathered}
\left(\frac{\partial u}{\partial y}+\frac{\partial v}{\partial x}\right)+\frac{1}{2}\left(\frac{d \sigma}{d x}+\frac{\lambda \rho^{s}}{\rho_{e}^{s}} \frac{d \rho_{e}^{s}}{d x}\right)=\bar{\beta} u, \\
v=Q\left(\rho^{s}-\rho_{e}^{s}\right) \\
\epsilon \frac{d\left(\rho^{s} v^{s}\right)}{d x}=-\left(\rho^{s}-\rho_{e}^{s}\right), \\
v^{s}=\frac{1}{2} u+\bar{\alpha}\left(\frac{d \sigma}{d x}+\frac{\lambda \rho^{s}}{\rho_{e}^{s}} \frac{d \rho_{e}^{s}}{d x}\right), \\
\sigma=\lambda\left(1-\rho^{s}\right), \\
\rho_{e}^{s}=\frac{1}{2}\left(\bar{\rho}_{1 e}^{s}+\bar{\rho}_{2 e}^{s}\right)+\frac{1}{2}\left(\bar{\rho}_{2 e}^{s}-\bar{\rho}_{1 e}^{s}\right) \tanh (x / \bar{l}),
\end{gathered}
$$

and in the far field

$$
u \rightarrow 1, v \rightarrow 0 \text { as } x^{2}+y^{2} \rightarrow \infty .
$$

Here

$$
\begin{gathered}
R e=\frac{\rho \sigma_{l g}^{2}}{S \mu^{3}}, \epsilon=S \tau, \bar{\beta}=\frac{\beta \sigma_{l g}}{\mu^{2} S}, Q=\frac{\rho_{(0)}^{s} \mu}{\rho \tau \sigma_{l g}}, \bar{\alpha}=\frac{\alpha S \mu^{2}}{\sigma_{l g}}, \\
\bar{l}=\frac{\mu S l}{\sigma_{l g}}, \lambda=\frac{\gamma \rho_{(0)}^{s}}{\sigma_{l g}}, \bar{\rho}_{i e}^{s}=\frac{\rho_{i e}^{s}}{\rho_{(0)}^{s}} \quad(i=1,2) .
\end{gathered}
$$

It is noteworthy that, unlike the classical Navier condition, in (12), which can be regarded as its generalisation, the tangential stress (the first term on the left-hand side) includes $\partial v / \partial x$. The deviation of $\rho^{s}$ from its equilibrium value and the resulting adsorption/desorption lead to $v \neq 0$ via (13). Then, the spatial non-uniformity of this process makes $\partial v / \partial x \neq 0$ thus 'switching on' this term in the tangential stress in (12) and hence contributing to the apparent slip. 
The parameter $Q$ in (13) is the ratio of the characteristic mass flux into (out of) the liquid-solid interface associated with the adsorption (desorption) process triggered by the deviation of the surface density from its local equilibrium value and the characteristic mass flux due to convection in the bulk, whereas $\epsilon^{-1}$ characterizes the ratio of the former and the characteristic divergence of the convective mass flux in the surface phase. In the present context, $\epsilon$ is simply the product of the magnitude of shear in the far field $S$ and the surface-tension-relaxation time $\tau$.

Given that it is the equilibrium contact angle $\theta$, which a liquid-gas free surface would form with a solid, that we use as a measure of the wettability of a solid substrate, it is convenient to eliminate $\bar{\rho}_{i e}^{s}$, which are 'internal' parameters of the model, in favour of $\theta_{i}$ using (7) and (16):

$$
\bar{\rho}_{i e}^{s}=1+\lambda^{-1} \cos \theta_{i} \quad(i=1,2) .
$$

Hereafter we will refer to the portion of the solid substrate with equilibrium contact angle $\theta_{1}$ and $\theta_{2}$ as 'solid 1 ' and 'solid 2', respectively.

Following from [25], where it was shown by analysing experiments on dynamic wetting that $\alpha \sim \beta^{-1}$, we will further assume that $\alpha \beta=1$ and hence that, in terms of our non-dimensional parameters, $\bar{\alpha} \bar{\beta}=1$. The analysis of experiments in [25] also provides estimates for the magnitude of phenomenological constants in the interface formation model's equations. Using these estimates and taking $\rho \sim 1 \mathrm{~g} \mathrm{~cm}^{-3}, \mu \sim 10^{-1}-10^{2} \mathrm{~g} \mathrm{~cm}^{-1} \mathrm{~s}^{-1}$, $\sigma_{l g} \sim 10-10^{2}$ dyn $\mathrm{cm}^{-1}, S \sim 10^{3}-10^{5} \mathrm{~s}^{-1}$ and $l \sim 10^{-6}-10^{-5} \mathrm{~cm}$ one arrives at a typical range of values for the magnitudes of the non-dimensional groups

$$
\begin{aligned}
& R e \sim 10^{-9}-10^{3}, \quad \epsilon \sim 10^{-6}-10^{-2}, \quad \bar{\beta} \sim 10^{1}-10^{7}, \\
& Q \sim 10^{-3}-10^{3}, \quad \bar{l} \sim 10^{-6}-10^{1}, \quad \lambda \sim 2-10^{2}, \\
& \theta_{i} \in\left[0^{\circ}, 180^{\circ}\right] \quad(i=1,2) \text {. }
\end{aligned}
$$

As mentioned earlier, it is the double limit $\bar{\beta}^{-1} \rightarrow 0, \epsilon \rightarrow$ 0 applied to equations (12)-(16) that results in the no-slip condition and hence all effects associated with deviation from the classical no-slip are at leading order in these parameters.

\section{SOLUTION}

The problem was solved numerically using the finite element method. Fig. 1 shows the streamlines of the flow for the case where solid 1 is more hydrophillic than solid 2 ; the values of the dimensionless constants are given in the figure caption. As one can see, when the outer flow drives the fluid from a hydrophillic to a hydrophobic zone, there appears a normal flux from the surface phase into the bulk. It is noteworthy that, as shown in Fig. 1] the vertical component of bulk velocity is nonzero at $y=0$, whereas for the classical Navier condition with different coefficients of sliding friction one has $v=0$ at $y=0$ [12] and the normal component of velocity away from the solid appears solely due to the disturbance of the tangential flow at $y=0$.

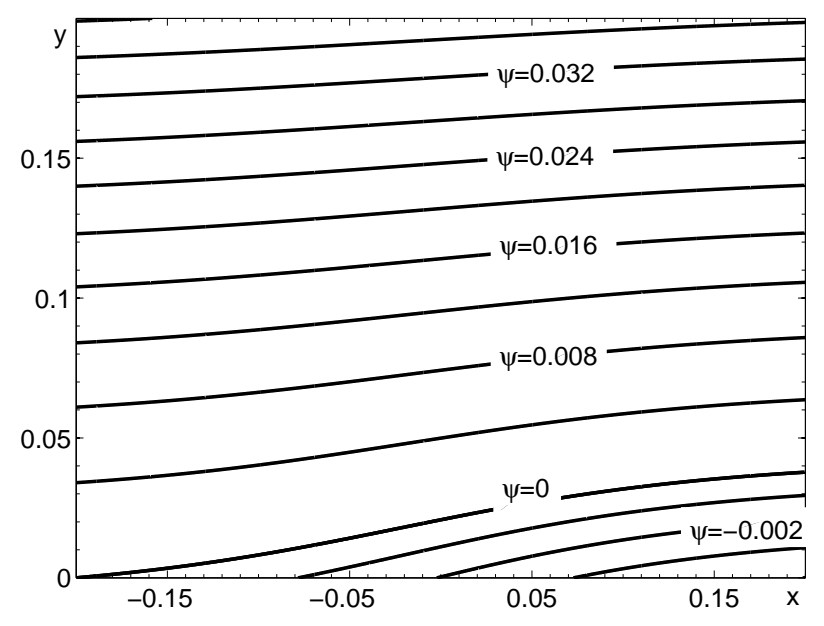

FIG. 1: Streamlines of flow over a chemically patterned surface in which values of the streamfunction $\psi$ are given. Parameter values $R e=0.01, \epsilon=0.01, \bar{\beta}=10, Q=100, \bar{l}=0.1$, $\lambda=10, \theta_{1}=10^{\circ}$ and $\theta_{2}=100^{\circ}$.

In Figs. 26 the distributions of the bulk velocity components along the solid surface are shown for the case in which solid 1 is more hydrophillic than solid 2. In each graph one can see (i) a positive normal velocity and (ii) variation in tangential velocity on the surface. The origin of the first of these effects is relatively straightforward. When fluid particles forming the interface are driven by the outer flow toward the region of lower equilibrium surface density, one has that in the disturbed equilibrium $\rho^{s}>\rho_{e}^{s}$, and hence, according to (13), $v>0$.

Importantly, it can be seen in Fig. 1 that the flux out of the surface phase occurs both in the hydrophillic $(x<0)$ and hydrophobic $(x>0)$ regions of the solid and extends itself well outside the transition zone. When solid 1 is more hydrophobic than solid 2 one observes the reverse effect, with the normal component of velocity directed towards the surface, corresponding to a flux into the surface phase. Once again this occurs on both sides of the transition region.

In order to understand the distribution of the tangential component of the bulk velocity on the surface we consider the terms on the left-hand side of the generalised Navier condition (12) which determine $u(x, 0)$. For a plane-parallel shear flow over a homogeneous surface, i.e. far away from the wettability transition region, there is no deviation of the surface density from its equilibrium value $\left(\rho^{s}=\rho_{e}^{s}\right)$ and hence no desorption from (or adsorption into) the liquid-solid interface $(v=0)$. This is an obvious solution of (11)-18). In dimensionless variables, the tangential stress on the surface, $\partial u / \partial y+\partial v / \partial x$, has magnitude $1(\partial u / \partial y=1, \partial v / \partial x=0)$. 


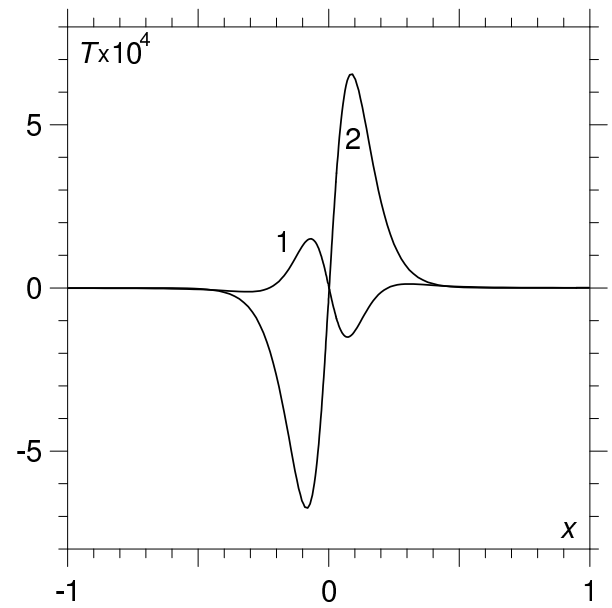

FIG. 2: Variation of the 'generators' of slip (the terms on the left hand side of the generalised Navier condition (12) in response to a variation in the solid surface wettability. In curve $1, \mathrm{~T}=[\partial u / \partial y+\partial v / \partial x-1]$ whilst in curve $2, \mathrm{~T}=\left[d \sigma / d x+\left(\lambda \rho^{s} / \rho_{e}^{s}\right) d \rho_{e}^{s} / d x\right] / 2$. Results are obtained for parameter values $R e=0.01, \epsilon=0.01, \bar{\beta}=100, Q=1$, $\bar{l}=0.1, \lambda=20, \theta_{1}=10^{\circ}, \theta_{2}=100^{\circ}$.

As one approaches the transition region, the situation changes. Curve 1 in Fig. 2 shows the distribution of the deviation of the tangential stress (the first term on the left-hand side in (12)) from the value 1 across this region. Importantly, there are now contributions to the tangential stress from both $\partial u / \partial y$ and $\partial v / \partial x$ since the latter becomes nonzero due to variation in the normal velocity along the surface as a result of the spatially nonuniform desorption (adsorption) process caused by the deviation of $\rho^{s}$ from its local equilibrium value. As discussed earlier, this effect could follow neither from the standard Navier condition nor from any of its generalizations if the interface formation process and the associated mass exchange between the interface and the bulk are not taken into account.

The other factors that, according to (12), lead to slip on the liquid-solid interface behave as follows. As mentioned in the previous section, in equilibrium the surface force, $\left[\left(\lambda \rho^{s} / \rho_{e}^{s}\right) d \rho_{e}^{s} / d x\right] / 2$, and the surface tension gradient, $(d \sigma / d x) / 2$, balance each other to ensure that there is no perpetual motion. However, when a shear flow in the far field is imposed, it disturbs the surface phase, and the above two terms are no longer in balance since they obviously depend differently on the variable surface density $\rho^{s}$. The deviation of the second term on the left-hand side of (12) from zero is shown as curve 2 in Fig. 2, One can see that it is the variation in this second term in (12) that dominates and hence it is the imbalance between the surface tension gradient and the tangential surface force caused by shear flow that is mainly responsible for a variation in slip on the surface as its wettability changes. The corresponding right-hand side of (12) and the normal velocity for these parameter values are shown by curve 3 of Fig. 3 .
Fig. [3illustrates the effect that the size of the parameters $\epsilon$ and $\bar{\beta}$ have on the solution. As was mentioned, all effects associated with deviations from no-slip, most clearly the nonzero normal velocity, are proportional to $\epsilon$ and $\bar{\beta}^{-1}$, and the results shown in the figure support this conclusion. Noticeably, the normal velocity on the surface appears to be symmetric about the centre of the transition region, where it achieves its maximum value. The tangential velocity seems to be antisymmetric about the origin, with a decrease in slip on solid 1 and increase in solid 2.

Fig. 4 shows the effect on the solution of the parameters $R e, Q$ and $\lambda$. Curves 2 and 3, which are almost indistinguishable, demonstrate that the Reynolds number has almost no effect on the dynamics of the surface. The local Reynolds number near the surface is always small due to the smallness of velocities in the vicinity of the solid surface even when the value associated with the global flow is relatively large, and one should indeed expect inertial effects to have little impact on dynamics in the surface phase.

The parameter $Q$ determines the degree of mass exchange between bulk and surface phase (13). Curves 2 and 4 in Fig. 4 show that the greater its value, the more pronounced the effect will be. By varying $\lambda$ one can see that the effect increases with the compressibility of the fluid.

Fig. 5 shows that for a smaller transition region one has a sharper effect. In all cases the disturbance, i.e. the non-zero normal component of the bulk velocity and variation in slip, runs well outside the region of varying wettability. The results suggest that if instead of a region, one treated the transition in wettability as a solidsolid-liquid contact line, then the disturbance to the bulk flow would be sharper than the effect associated with a finite transition region but would still occur over a finite region of the interface.

It is interesting to note that although the shape of the curves in Fig. 5 differ, the integral of the normal velocity, i.e. the total flux out of the surface phase per unit time

$$
J=\int_{-\infty}^{\infty} v d x
$$

which we consider as a measure of the effect that a patterned surface has on an adjacent flow, remains unchanged. The value of $J$ is a sensible choice of measure as we have seen that it is the normal component of velocity that causes the noticeable deviation from plane-parallel shear flow.

Given that the change in solid does indeed alter the flow of an adjacent liquid, consider how the magnitude of the effect is dependent on the choice of solids. This can be illustrated by looking at three different solids characterised by contact angles of $10^{\circ}, 60^{\circ}$ and $110^{\circ}$ that a free surface would form with them. Fig. 6 shows the velocity components for three different combinations of solids for the case where the first solid is more hydrophillic. The results suggest that the normal flux per unit time 

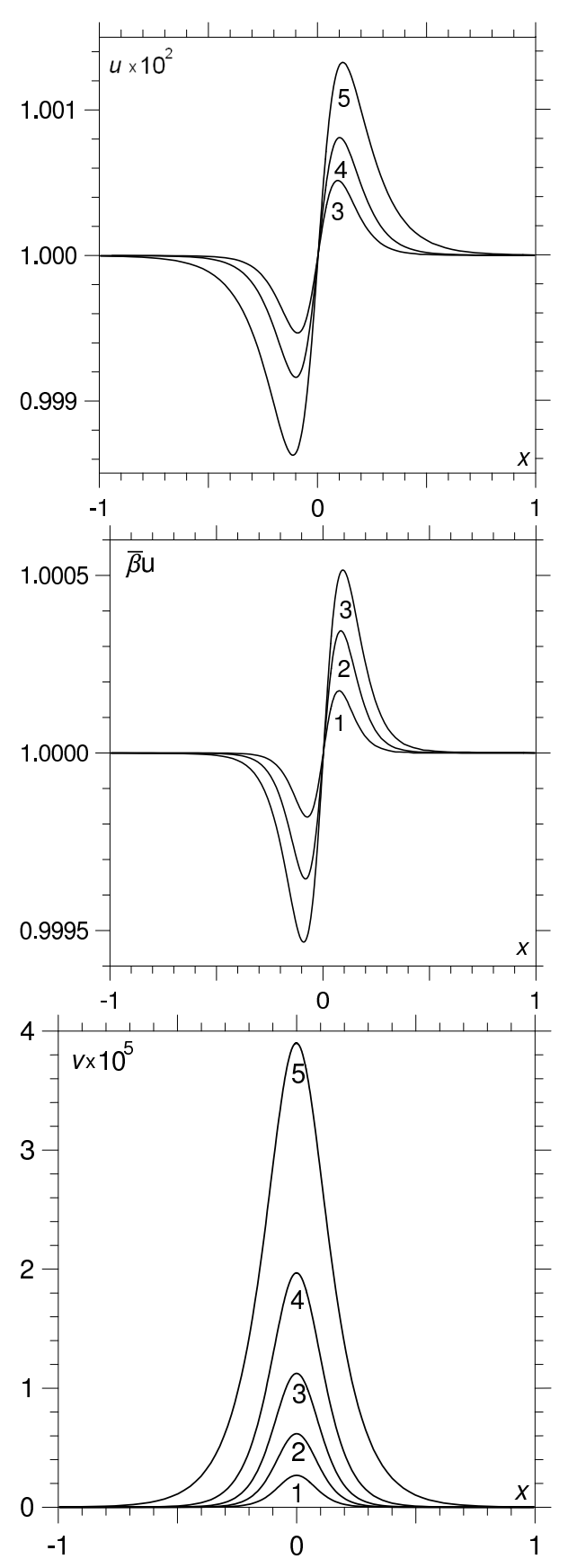

FIG. 3: Distribution of $u(x, 0)$ and $v(x, 0)$ for different $\epsilon$ and $\bar{\beta}$. Curves $1-5$ correspond to $\epsilon=0.01, \bar{\beta}=500 ; \epsilon=0.01$, $\bar{\beta}=200 ; \epsilon=0.01, \bar{\beta}=100 ; \epsilon=0.02, \bar{\beta}=100 ; \epsilon=0.05$, $\bar{\beta}=100$, respectively; for all curves $R e=0.01, Q=1, \bar{l}=0.1$, $\lambda=20, \theta_{1}=10^{\circ}$ and $\theta_{2}=100^{\circ}$.

is proportional to the difference $\cos \theta_{1}-\cos \theta_{2}$ which explains the ordering in Fig. 6] where we can see the case $10^{\circ} \rightarrow 60^{\circ}$ gives a far smaller effect than $60^{\circ} \rightarrow 110^{\circ}$ despite the difference in contact angles being the same. The numerical analysis of the problem made it possible to advance and then verify the following approximate formula

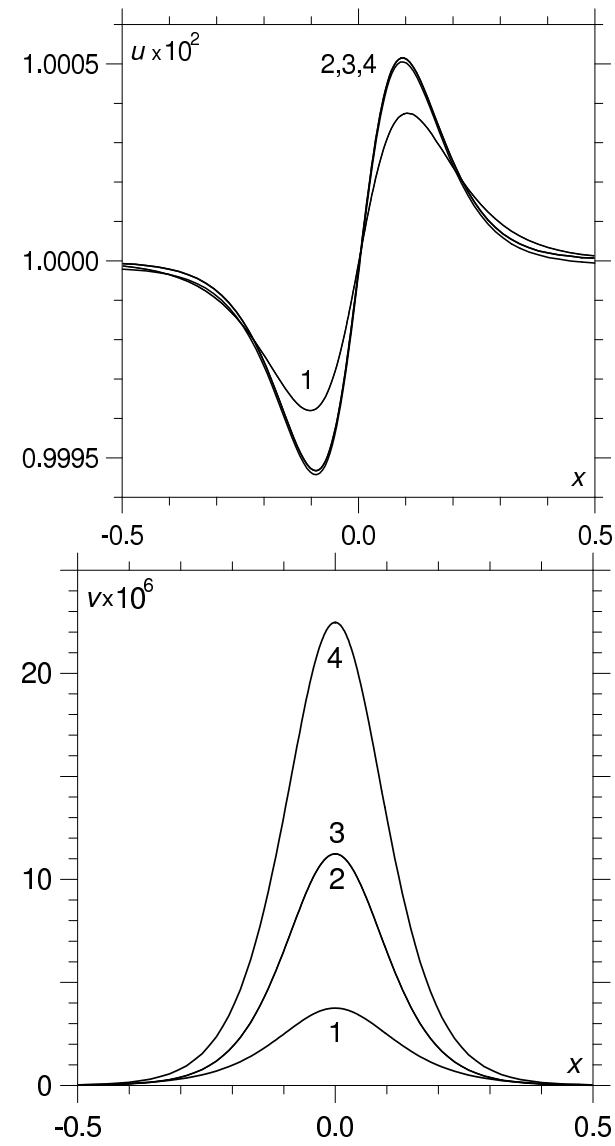

FIG. 4: Dependence of $u(x, 0)$ and $v(x, 0)$ on $R e, Q$ and $\lambda$. Curve 2 corresponds to $\operatorname{Re}=0.01, Q=1, \lambda=20 ; 1: \lambda=50$; 3 (indistinguishable within the graphical accuracy): $R e=10$; 4: $Q=2$. Parameters $\epsilon=0.01, \bar{\beta}=100, \bar{l}=0.1, \theta_{1}=10^{\circ}$, $\theta_{2}=100^{\circ}$ for all curves.

for $J$ :

$$
J=\frac{\epsilon Q}{2 \bar{\beta} \lambda}\left(\cos \theta_{1}-\cos \theta_{2}\right) .
$$

This formula, which very accurately represent the numerical data over a wide range of parameter values, has been obtained as follows. The proportionality of the flux between the surface and bulk phases to the cosines of the contact angle is in fact what one should expect given that according to (6) and (77), $\cos \theta$ is a linear function of the equilibrium surface density, and it is the difference $\bar{\rho}_{1 e}^{s}-\bar{\rho}_{2 e}^{s}$ that determines the flux. This conjecture of the linear dependence of $J$ on $\cos \theta_{1}-\cos \theta_{2}$ is the only nontrivial step in obtaining (21) and it has to be verified numerically. We also have to find the coefficient of proportionality in this dependence in terms of the dimensionless parameters of the problem. In order to do this, we used the following procedure. For the fixed values of $\theta_{1}$ and $\theta_{2}$, we chose a base state in terms of the remaining parameters and then varied one of these parameters away from its base state to consider the effect this variation produces on $J$. After repeating this operation for 


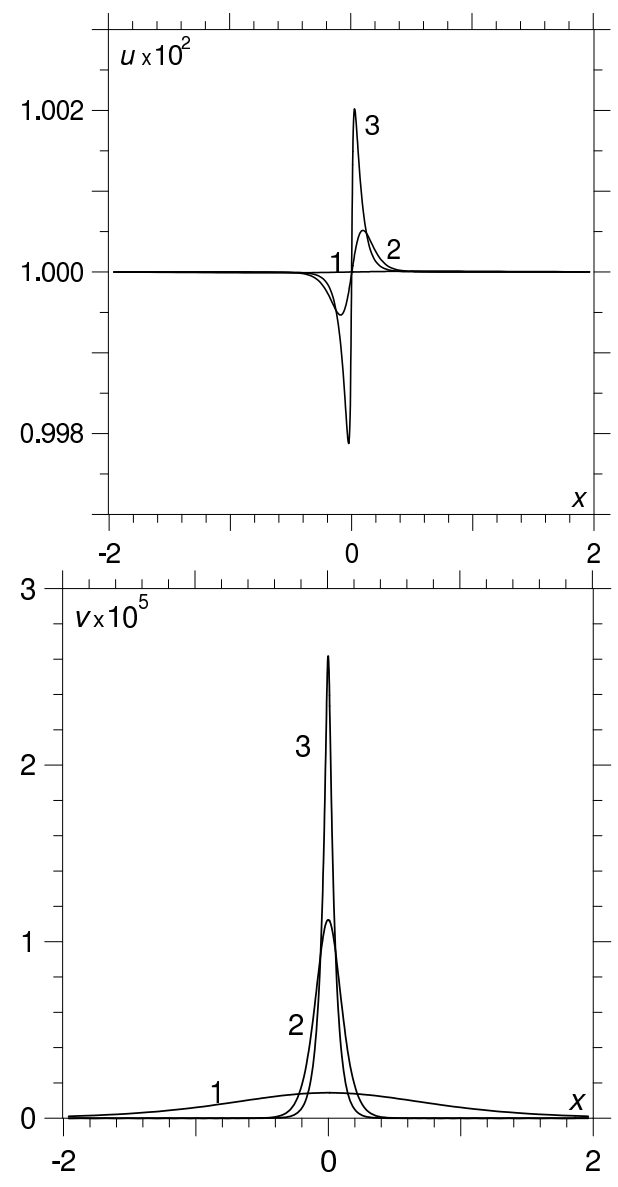

FIG. 5: Velocity on the surface for different widths of transition region. Curves $1-3$ correspond to $\bar{l}=1, \bar{l}=0.1$ and $\bar{l}=0.01$, respectively; for all curves $R e=0.01, \epsilon=0.01$, $\bar{\beta}=100, Q=1, \lambda=20, \theta_{1}=10^{\circ}$ and $\theta_{2}=100^{\circ}$.

all parameters we arrive at equation (21) which now has to be verified by independently varying all dimensionless parameters, including $\theta_{1}$ and $\theta_{2}$. The accuracy with which this equation represents $J$ is illustrated in Fig. 7

Order of magnitude arguments for the phenomenological parameters and the analysis of experiments on dynamic wetting [25] suggest that $\beta \sim \mu / h$, where $h$ is the thickness of the interfacial layer (modelled here as an 'interface' of zero thickness). Using this estimate, for the dimensional flux per unit time out of the surface phase in a liquid/solid/solid system one has

$$
J_{d i m} \sim \frac{S h \sigma_{l g}}{\rho \gamma}\left(\cos \theta_{1}-\cos \theta_{2}\right) .
$$

Given that $h$ is typically very small (a few $\mathrm{nm}$ for simple fluids [26]), the above estimate highlights the subtle nature of the effects that we have described.

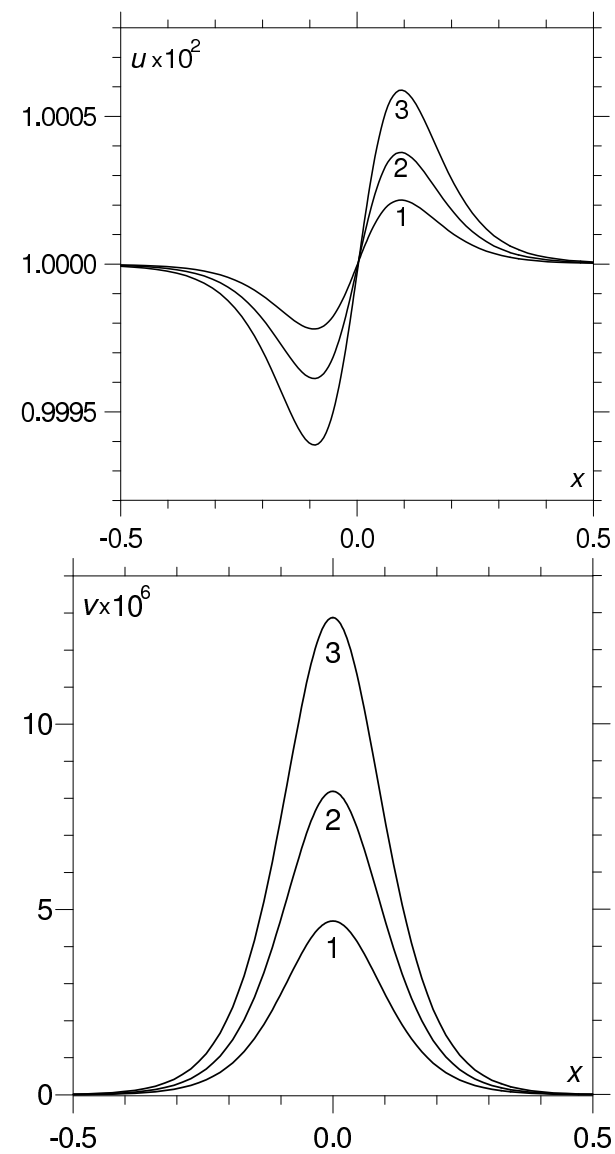

FIG. 6: Velocity on the surface for different solid-solid pairs. Curve 1: $\theta_{1}=10^{\circ}, \theta_{2}=60^{\circ} ; 2: \theta_{1}=60^{\circ}, \theta_{2}=110^{\circ} ; 3$ : $\theta_{1}=10^{\circ}, \theta_{2}=110^{\circ}$, respectively. For all curves $R e=0.01$, $\epsilon=0.01, \bar{\beta}=100, Q=1, \bar{l}=0.1, \lambda=20$.

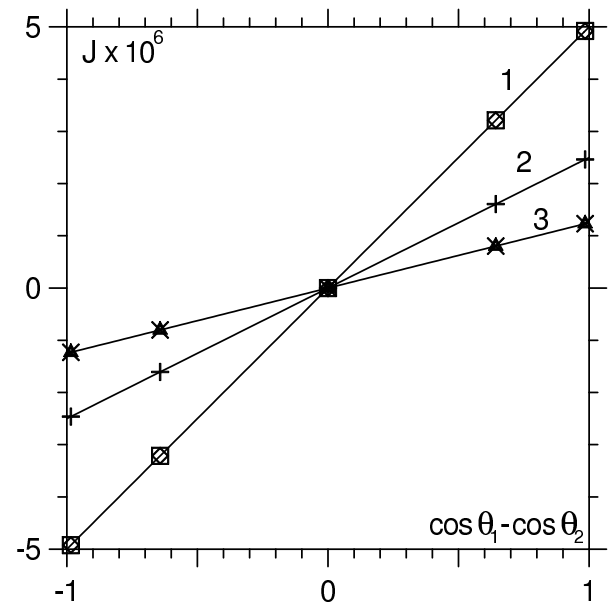

FIG. 7: Dependence of flux on parameters $Q, \epsilon, \bar{\beta}$ and $\lambda$. Parameters are varied around a base state, the results of which are represented by vertical crosses, of: $\bar{\beta}=100, Q=1, \epsilon=$ $0.01, \lambda=20$. Then, diamonds: $\epsilon=0.02$; squares: $Q=2$; triangles: $\bar{\beta}=200$; diagonal crosses: $\lambda=40$. For all curves $\theta_{1}=90^{\circ}$ whilst $\theta_{2}$ is varied, and $R e=0.01$. Curves $1-3$ represent the predicted flux given by (21). 


\section{CONCLUSION}

As was shown, the interface formation model applied to the flow over a solid surface of variable wettability allows one to describe the main features of this flow observed in molecular dynamics simulations, most notably the nonzero component of the bulk velocity normal to the solid surface. A natural link between 'wettability' interpreted in terms of the concept of the 'contact angle' featuring in the spreading of liquids on solid surfaces and a viscous flow over chemically-patterned solids with no free surface present has been established. Importantly, the interface formation model deals with these phenomena entirely within the approach of continuum mechanics with no artificial inclusion of intermolecular forces in its framework. An interesting feature that follows from the results is that slip, i.e. the difference between the tangential component of the fluid's velocity and the corresponding component of the velocity of the solid surface, results primarily from the disturbance of the force balance in the 'surface phase' and not from the tangential stress, as follows from the standard Navier condition.

\section{Acknowledgments}

The authors kindly acknowledge the financial support of Kodak European Research and the EPSRC via a Mathematics CASE award.
[1] Y. Xia, D. Qin, and Y. Yin, Current Opinion Coll. \& Int. Sci 6, 54 (2001).

[2] A. Darhuber and S. Troian, Annu. Rev. Fluid Mech. 37, 425 (2005).

[3] T. Squires and S. Quake, Rev. Mod. Phys. 77, 977 (2005).

[4] B. Zhao, J. Moore, and D. Beebe, Science 291, 1023 (2001).

[5] H. Gau, S. Herminghaus, P. Lenz, and R. Lipowsky, Science 283, 46 (1999).

[6] S. Burns, P. Cain, J. Mills, J. Wang, and H. Sirringhaus, MRS Bulletin 28, 829 (2003).

[7] A. Dupuis and J. Yeomans, Future generation computer systems 20, 993 (2004).

[8] H. Braun and E. Meyer, Thin Solid Films 345, 222 (1999).

[9] M. Chaudhury and G. Whitesides, Science 256, 1539 (1992).

[10] S. Daniel, M. Chaudhury, and J. Chen, Science 291, 633 (2001).

[11] N. Priezjev, A. Darhuber, and S. Troian, Phys. Rev. E 71, 041608 (2005).

[12] T. Qian, X. Wang, and P. Sheng, Phys. Rev. E 72,
022501 (2005).

[13] E. Dussan and S. Davis, J. Fluid Mech. 65, 71 (1974).

[14] E. Dussan, Annu. Rev. Fluid Mech 11, 371 (1979).

[15] C. Navier, Mém. de l'Acad. de Sciences l'Inst. de France 6, 389 (1823).

[16] H. Lamb, Hydrodynamics (Dover, New York, 1932).

[17] E. Lauga, M. Brenner, and H. Stone, Handbook of experimental fluid dynamics (New York: Springer, 2005).

[18] P. Thompson and S. Troian, Nature 389, 360 (1997).

[19] V. Sokhan, D. Nicholson, and N. Quirke, J. Chem. Phys. 115, 3878 (2001).

[20] Y. Shikhmurzaev, J. Fluid Mech. 334, 211 (1997).

[21] Y. Shikhmurzaev, Int. J. Multiphase Flow 19, 589 (1993).

[22] Y. Shikhmurzaev, Phys. Lett. A 345, 378 (2005).

[23] Y. Shikhmurzaev, IMA J. Appl. Maths 70, 880 (2005).

[24] Y. Shikhmurzaev, C.R. Mecanique 333, 205 (2005).

[25] T. Blake and Y. Shikhmurzaev, J. Coll. Int. Sci. 253, 196 (2002).

[26] J. Rowlinson and B. Widom, Molecular Theory of Capillarity (Clarendon Press, Oxford, 1982). 